\title{
DIFFERENCES BETWEEN PERFECT POWERS
}

\author{
MICHAEL A. BENNETT
}

ABSTRACT. We apply the hypergeometric method of Thue and Siegel to prove, if $a$ and $b$ are positive integers, that the inequality

$$
0<\left|a^{x}-b^{y}\right|<\frac{1}{4} \max \left\{a^{x / 2}, b^{y / 2}\right\}
$$

has at most a single solution in positive integers $x$ and $y$. This essentially sharpens a classic result of LeVeque.

\section{INTRODUCTION}

In 1950, LeVeque [10] proved, given fixed positive integers $a$ and $b$, that the Diophantine equation

$$
a^{x}-b^{y}=1
$$

has at most a single solution in positive integers $x$ and $y$, unless $(a, b)=(3,2)$, in which case two such solutions accrue. Nowadays, this might be regarded as a very special case of the profound work of Mihailescu [11] on Catalan's conjecture, but, in fairness, one should note that [10] inspired work of Cassels ([4] and [5]) which, in turn, proved crucial to Mihailescu.

If one considers more general equations of the shape

$$
a^{x}-b^{y}=c
$$

where $c>1$ is fixed, then no conclusion of even remotely comparable strength to those in [11] is available to us. If, in analogy to LeVeque [10], we assume that a and $b$ are fixed, however, then equation (1.1) has at most two solutions in positive integers $(x, y)$ (see the author's [2] and earlier work of Herschfeld [7], Pillai [12], [13], [14], [15]). Recently, this result has been extended to equations of the shape

$$
\left|a^{x} \pm b^{y}\right|=c,
$$

by Scott and Styer [17].

The goal of this paper is a broad generalization of the main theorem of [10], where, instead of a Diophantine equation, we consider a corresponding Diophantine inequality.

Theorem 1.1. Let $a$ and $b$ be positive integers. Then there exists at most one pair of positive integers $(x, y)$ for which

$$
0<\left|a^{x}-b^{y}\right|<\frac{1}{4} \max \left\{a^{x / 2}, b^{y / 2}\right\} .
$$

Received by the editors April 14, 2006.

1991 Mathematics Subject Classification. Primary 11D61, 11D45.

Supported in part by a grant from NSERC. 
It should be noted that lower bounds for linear forms in logarithms may be used to show that there are in fact no solutions whatsoever to (1.2), provided $x \geq x_{0}(a, b)$ (see Ellison [6]; more recent work of Laurent, Mignotte and Nesterenko [9] may be used to sharpen this result), which leads to an alternative proof of Theorem 1.1, for sufficiently large $a$ and $b$. Our proof, in contrast, will rely upon the hypergeometric method of Thue-Siegel which, to our knowledge, has not been applied previously in this context.

Theorem 1.1 leads rather easily to a sharpening of the results of [2] and [17]; we will not undertake this here.

\section{ElEMENTARY PRELIMINARIES}

We will suppose, here and henceforth, that $a$ and $b$ are positive integers, and that $\left(x_{1}, y_{1}\right)$ and $\left(x_{2}, y_{2}\right)$ are two solutions in positive integers to inequality (1.2) with, say, $x_{2}>x_{1}$. Without loss of generality, we may assume that neither $a$ nor $b$ is a perfect power. Let us write

$$
a^{x_{i}}-b^{y_{i}}=c_{i},
$$

where, by symmetry, we may assume that $c_{1}>0$. For future use, it will prove convenient to note that

$$
\min \left\{a^{x_{i}}, b^{y_{i}}\right\}>\frac{15}{16} \max \left\{a^{x_{i}}, b^{y_{i}}\right\}
$$

To see this, observe that the inequality

$$
\min \left\{a^{x_{i}}, b^{y_{i}}\right\} \leq \frac{15}{16} \max \left\{a^{x_{i}}, b^{y_{i}}\right\}
$$

implies

whereby, from (1.2),

$$
\left|c_{i}\right| \geq \frac{1}{16} \max \left\{a^{x_{i}}, b^{y_{i}}\right\}
$$

$$
\frac{1}{16} \max \left\{a^{x_{i}}, b^{y_{i}}\right\}<\frac{1}{4} \max \left\{a^{x_{i}}, b^{y_{i}}\right\}^{1 / 2},
$$

and so $\max \left\{a^{x_{i}}, b^{y_{i}}\right\}<16$, contradicting (1.2) and the fact that $\left|c_{i}\right| \geq 1$.

Next, let us show that necessarily $x_{i}$ and $y_{i}$ are coprime. If we suppose

$$
\operatorname{gcd}\left(x_{i}, y_{i}\right)=d>1
$$

and write $x_{i}=x_{0} d, y_{i}=y_{0} d$, then, from (2.1) and the fact that $a^{x_{i}} \neq b^{y_{i}}$ (whereby $\left.\left|a^{x_{0}}-b^{y_{0}}\right| \geq 1\right)$, we have

$$
\left|c_{i}\right| \geq d \min \left\{a^{x_{0}(d-1)}, b^{y_{0}(d-1)}\right\}=d \min \left\{a^{x_{i}}, b^{y_{i}}\right\}^{(d-1) / d},
$$

and so

$$
d \min \left\{a^{x_{i}}, b^{y_{i}}\right\}^{(d-1) / d}<\frac{1}{4} \max \left\{a^{x_{i}}, b^{y_{i}}\right\}^{1 / 2} .
$$

Applying inequality (2.2), it follows that

whereby

$$
d \min \left\{a^{x_{i}}, b^{y_{i}}\right\}^{(d-1) / d}<\frac{1}{\sqrt{15}} \min \left\{a^{x_{i}}, b^{y_{i}}\right\}^{1 / 2},
$$

contradicting $d \geq 2$.

$$
\min \left\{a^{x_{i}}, b^{y_{i}}\right\}^{\frac{1}{2}-\frac{1}{d}}<\frac{1}{d \sqrt{15}}
$$




\section{A GAP PRINCIPLE}

As is rather standard when counting solutions to Diophantine equations or inequalities, we will require a result which guarantees that the putative solutions $\left(x_{1}, y_{1}\right)$ and $\left(x_{2}, y_{2}\right)$ to $(1.2)$ are of very different size. To derive this, we will begin with equation (2.1) which, after dividing by $b^{y_{i}}$ becomes

$$
a^{x_{i}} b^{-y_{i}}-1=c_{i} b^{-y_{i}} .
$$

Examination of the Maclaurin series for $e^{z}$ thus shows that

$$
\left|x_{1} \log a-y_{1} \log b\right|<c_{1} b^{-y_{1}}
$$

and

$$
\left|x_{2} \log a-y_{2} \log b\right|<2\left|c_{2}\right| b^{-y_{2}}
$$

(recall that $\left.c_{1}>0\right)$. Thus

$$
\left|\frac{\log b}{\log a}-\frac{x_{i}}{y_{i}}\right|<\frac{2^{i-1}\left|c_{i}\right|}{y_{i} b^{y_{i}} \log a}
$$

whereby we may conclude that $x_{i} / y_{i}$ is a convergent in the simple continued fraction expansion to $\frac{\log b}{\log a}$, provided, say,

$$
\frac{b^{y_{i}} \log a}{\left|c_{i}\right| y_{i}}>4 \geq 2^{i}
$$

Now, from (1.2) and (2.2), we have that

$$
\frac{b^{y_{i}} \log a}{\left|c_{i}\right| y_{i}}>\frac{\sqrt{15} b^{y_{i} / 2} \log a}{y_{i}} .
$$

If $a=2$ then $b \geq 3$ and hence $b^{y_{i} / 2} / y_{i} \geq 3 / 2$, while, if $a \geq 3, b^{y_{i} / 2} / y_{i} \geq 2 \sqrt{2} / 3$. In both cases, inequality (3.2) obtains.

It follows, therefore, that $x_{i} / y_{i}$ is a convergent in the simple continued fraction expansion to $\frac{\log b}{\log a}$ for both $i=1$ and $i=2$. On the other hand, if $p_{n} / q_{n}$ is the $n$th such convergent, then

$$
\left|\frac{\log b}{\log a}-\frac{p_{n}}{q_{n}}\right|>\frac{1}{\left(a_{n+1}+2\right) q_{n}^{2}}
$$

where $a_{n+1}$ is the $(n+1)$ st partial quotient to $\frac{\log b}{\log a}$ (see e.g. [8]). Since

$$
\operatorname{gcd}\left(x_{1}, y_{1}\right)=\operatorname{gcd}\left(x_{2}, y_{2}\right)=1,
$$

it follows, if $x_{1} / y_{1}=p_{r} / q_{r}$ and $x_{2} / y_{2}=p_{s} / q_{s}$, that

$$
x_{1}=p_{r}, y_{1}=q_{r}, x_{2}=p_{s} \text { and } y_{2}=q_{s} .
$$

Combining (3.1) and (3.3) thus yields

$$
a_{r+1}>\frac{b^{y_{1}} \log a}{c_{1} y_{1}}-2
$$

and, since $p_{s} \geq p_{r+1}>a_{r+1} p_{r}$,

$$
x_{2}>\left(\frac{b^{y_{1}} \log a}{c_{1} y_{1}}-2\right) x_{1} .
$$


From (1.2) and (2.2), we thus have that

$$
x_{2}>\left(\frac{\sqrt{15} b^{y_{1} / 2} \log a}{y_{1}}-2\right) x_{1} .
$$

Similarly, we obtain the inequality

$$
a_{s+1}>\frac{b^{y_{2}} \log a}{2\left|c_{2}\right| y_{2}}-2>\frac{\sqrt{15} b^{q_{s} / 2} \log a}{2 q_{s}}-2 .
$$

\section{Some Useful Polynomials}

Our main tool in proving Theorem 1.1 will be (off-diagonal) Padé approximants to binomial functions of the shape $(1-z)^{k}$. We will generate these as in [1] (see also [3]). Let $A, B$ and $C$ be positive integers and define

$$
\begin{gathered}
P_{A, B, C}(z)=\frac{(A+B+C+1) !}{A ! B ! C !} \int_{0}^{1} u^{A}(1-u)^{B}(z-u)^{C} d u \\
Q_{A, B, C}(z)=\frac{(-1)^{C}(A+B+C+1) !}{A ! B ! C !} \int_{0}^{1} u^{B}(1-u)^{C}(1-u+z u)^{A} d u
\end{gathered}
$$

and

$$
E_{A, B, C}(z)=\frac{(A+B+C+1) !}{A ! B ! C !} \int_{0}^{1} u^{A}(1-u)^{C}(1-z u)^{B} d u .
$$

Arguing as in Section 2 of [1], we find that

$$
P_{A, B, C}(z)-(1-z)^{B+C+1} Q_{A, B, C}(z)=z^{A+C+1} E_{A, B, C}(z) .
$$

It is worth observing that if $A=C$, then $P_{A, B, C}(z)$ and $Q_{A, B, C}(z)$ correspond to the diagonal Padé approximants to $(1-z)^{B+C+1}$ with error term $E_{A, B, C}(z)$. The following results are given in [1] and [3]:

Lemma 4.1. The expressions $P_{A, B, C}(z), Q_{A, B, C}(z)$ and $E_{A, B, C}(z)$ satisfy

$$
\begin{aligned}
& P_{A, B, C}(z)=\sum_{r=0}^{C}\left(\begin{array}{c}
A+B+C+1 \\
r
\end{array}\right)\left(\begin{array}{c}
A+C-r \\
A
\end{array}\right)(-z)^{r}, \\
& Q_{A, B, C}(z)=(-1)^{C} \sum_{r=0}^{A}\left(\begin{array}{c}
A+C-r \\
C
\end{array}\right)\left(\begin{array}{c}
B+r \\
r
\end{array}\right) z^{r}
\end{aligned}
$$

and

$$
E_{A, B, C}(z)=\sum_{r=0}^{B}\left(\begin{array}{c}
A+r \\
r
\end{array}\right)\left(\begin{array}{c}
A+B+C+1 \\
A+C+r+1
\end{array}\right)(-z)^{r} .
$$

Lemma 4.2. There is a non-zero integer $D=D(A, B)$ for which

$$
P_{A, B, A}(z) Q_{A+1, B-1, A+1}(z)-Q_{A, B, A}(z) P_{A+1, B-1, A+1}(z)=D z^{2 A+1} .
$$

In summary, Lemma 4.1 implies that $P_{A, B, C}(z), Q_{A, B, C}(z)$ and $E_{A, B, C}(z)$ are polynomials in $z$ with integer coefficients, while Lemma 4.2 ensures that

$$
\left(P_{A, B, A}(z), P_{A+1, B-1, A+1}(z)\right) \text { and }\left(Q_{A, B, A}(z), Q_{A+1, B-1, A+1}(z)\right)
$$

are pairs of relatively prime polynomials. 


\section{Bounding the Approximants}

For our purposes, we will need to find reasonably sharp upper bounds upon the approximating polynomials defined in the previous section, viz

Lemma 5.1. If $n=m-\delta$ for $\delta \in\{0,1\}$ and $0<z<1 / 2$, then

$$
\left|P_{n}(z)\right|<\frac{4 \sqrt{2}}{3 \pi} \cdot 4^{m} \text { and }\left|E_{n}(z)\right|<\frac{4}{3 \sqrt{2} \pi} \cdot 16^{m} \text {. }
$$

Proof. We take $A=C=n=m-\delta$ and $B=3 m-n-1=2 m+\delta-1$ and begin by noting that a routine application of Stirling's formula yields the inequality

$$
\frac{(4 m) !}{(m !)^{2}(2 m) !}<\frac{1}{\sqrt{2} \pi m} \cdot 64^{m}
$$

valid for all positive integers $m$. It follows from (4.1), if we define

$$
u_{1}=\frac{1}{8}\left(3 z+2+\sqrt{4-4 z+9 z^{2}}\right)
$$

and

$$
P(z)=u_{1}\left(1-u_{1}\right)^{2}\left(z-u_{1}\right)
$$

that

$$
\left|P_{n}(z)\right|<\frac{\sqrt{2}}{8^{\delta} \pi} \cdot 64^{m}|P(z)|^{m-1}\left|\int_{0}^{1} u^{1-\delta}(1-u)^{1+\delta}(z-u)^{1-\delta} d u\right| .
$$

Via calculus, it is easy to show that $|P(z)|<1 / 16$, for $0<z<1 / 2$. Also

$$
\left|\int_{0}^{1} u(1-u)(z-u) d u\right|=\frac{1}{12}-\frac{z}{6}<\frac{1}{12}
$$

and

and hence the bound for $\left|P_{n}(z)\right|$ follows.

$$
\int_{0}^{1}(1-u)^{2} d u=\frac{1}{3}
$$

Similarly, if we define

$$
u_{2}=\frac{1}{8 z}\left(3 z+2-\sqrt{4-4 z+9 z^{2}}\right)
$$

and

$$
E(z)=u_{2}\left(1-u_{2}\right)\left(1-z u_{2}\right)^{2}
$$

then

$$
\left|E_{n}(z)\right|<\frac{\sqrt{2}}{8^{\delta} \pi} \cdot 64^{m}|E(z)|^{m-1}\left|\int_{0}^{1} u^{1-\delta}(1-u)^{1-\delta}(1-z u)^{1+\delta} d u\right| .
$$

Once again, it is easy to show that $|E(z)|<1 / 4$, for $0<z<1 / 2$, and that

$$
\left|\int_{0}^{1} u(1-u)(1-z u) d u\right|=\frac{1}{6}-\frac{z}{12}<\frac{1}{6}
$$

and

which leads to the desired result.

$$
\int_{0}^{1}(1-z u)^{2} d u=1-z+\frac{z^{2}}{3}<1
$$


Lemma 5.1 provides us with archimedean bounds for our approximants. Regarding non-archimedean information, let us define

$$
G(n)=\underset{r \in\{0,1, \ldots, n\}}{\operatorname{gcd}}\left(\left(\begin{array}{c}
2 n-r \\
n
\end{array}\right)\left(\begin{array}{c}
3 m-n-1+r \\
r
\end{array}\right)\right) .
$$

If we take $n=m$ or $m-1$, it follows from Lemma 7 of [1] that

$$
\lim _{m \rightarrow \infty} \frac{1}{m} \log G(n)=\frac{\pi}{2}-3 \log 2,
$$

and hence there exists a constant $c$ such that, for $n=m$ or $m-1$, and $m \geq 1$,

$$
G(n)>c \cdot 1.663^{m}
$$

For our purposes, we will have need of a completely explicit result along these lines; the proof of this follows arguments sketched on page 200 of [1] and relies upon Chebyshev-type estimates for primes in intervals.

Proposition 5.2. If $m$ is a positive integer and $n=m$ or $m-1$, then

$$
G(n)>0.00279 \cdot 1.5498^{m} \text {. }
$$

We note that we could avoid use of this proposition if we were prepared to treat certain "small" cases of Theorem 1.1 via lower bounds for linear forms in logarithms.

\section{The Proof of Theorem 1.1}

To proceed with the proof of Theorem 1.1, let us begin by writing

$$
x_{2}=3 x_{1} m+\alpha \text { and } y_{2}=3 y_{1} m^{\prime}+\beta,
$$

where

$$
0 \leq \alpha<3 x_{1} \text { and } 0 \leq \beta<3 y_{1}
$$

so that

$$
c_{2}=a^{3 x_{1} m} M_{1}-b^{3 y_{1} m^{\prime}} M_{2}
$$

with $M_{1}=a^{\alpha}$ and $M_{2}=b^{\beta}$. We claim that $m^{\prime} \geq m$. If not, then

$$
a^{x_{2}}-b^{y_{2}} \geq a^{3 x_{1} m_{2}} \cdot a^{3 x_{1}+\alpha}-b^{3 y_{1} m^{\prime}} \cdot b^{\beta}>a^{3 x_{1} m^{\prime}} \cdot a^{3 x_{1}}-b^{3 y_{1} m^{\prime}} \cdot b^{3 y_{1}}
$$

and so

$$
a^{x_{2}}-b^{y_{2}}>b^{3 y_{1}}\left(\left(a^{x_{1}}\right)^{3 m^{\prime}}-\left(b^{y_{1}}\right)^{3 m^{\prime}}\right) .
$$

It follows that either $m^{\prime}=0$ (so that $0 \leq y_{2}<3 y_{1}$, contradicting the combination of $(2.2)$ and $(3.5))$ or that $3 m^{\prime} \geq 3$. In the latter case, we have

$$
\left(a^{x_{1}}\right)^{3 m^{\prime}}-\left(b^{y_{1}}\right)^{3 m^{\prime}}>c_{1} \cdot 3 m^{\prime} \cdot\left(b^{y_{1}}\right)^{3 m^{\prime}-1}
$$

whence

$$
c_{2}=a^{x_{2}}-b^{y_{2}}>c_{1} \cdot 3 m^{\prime} \cdot\left(b^{y_{1}}\right)^{3 m^{\prime}+1}>b^{y_{2}},
$$

a contradiction. It follows that we may write

$$
a^{3 x_{1} m} M_{1}-b^{3 y_{1} m} M_{3}=c_{2},
$$

with $M_{3}=b^{\beta+3 y_{1}\left(m^{\prime}-m\right)}$.

We take $n=m$ or $m-1$. Here and subsequently, let $A=C=n, B=3 m-n-1$ and write, suppressing various dependencies,

$$
P_{n}(z)=P_{n, 3 m-n-1, n}(z), \quad Q_{n}(z)=Q_{n, 3 m-n-1, n}(z),
$$


and

$$
E_{n}(z)=E_{n, 3 m-n-1, n}(z) .
$$

Fixing once and for all $z=z_{0}=c_{1} / a^{x_{1}}$ and substituting this into (4.4), we find that

$$
a^{3 x_{1} m} P-b^{3 y_{1} m} Q=E
$$

where

and

$$
P=\frac{1}{G(n)} a^{x_{1} n} P_{n}\left(z_{0}\right), \quad Q=\frac{1}{G(n)} a^{x_{1} n} Q_{n}\left(z_{0}\right)
$$

$$
E=\frac{1}{G(n)}\left(a^{x_{1}}\right)^{3 m-n-1} c_{1}^{2 n+1} E_{n}\left(z_{0}\right) .
$$

It follows that $P, Q$ and $E$ are all integers. Multiplying (6.1) by $P$ and (6.2) by $M_{1}$, we deduce the inequality

$$
b^{3 y_{1} m}\left|M_{3} P-M_{1} Q\right| \leq\left|c_{2}\right||P|+|E| M_{1} .
$$

We claim that for at least one of $n=m$ or $n=m-1$, say $n=m-\delta$, we have $M_{3} P \neq M_{1} Q$. Indeed, if this fails to be the case, then

$$
M_{3} P_{m-1}\left(z_{0}\right)=M_{1} Q_{m-1}\left(z_{0}\right) \text { and } M_{3} P_{m}\left(z_{0}\right)=M_{1} Q_{m}\left(z_{0}\right),
$$

whereby

$$
P_{m-1}\left(z_{0}\right) Q_{m}\left(z_{0}\right)=Q_{m-1}\left(z_{0}\right) P_{m}\left(z_{0}\right),
$$

contradicting Lemma 4.2. For this $n=m-\delta$, we therefore have

$$
b^{3 y_{1} m} \leq\left|c_{2}\right||P|+|E| M_{1} .
$$

To proceed, we will show that each of $|P|$ and $|E|$ is not too large, whereby we may employ (6.3) to obtain a (typically contradictory) lower bound on $M_{1}$.

Let us begin by showing that

$$
b^{3 y_{1} m}>31\left|c_{2}\right||P| .
$$

We will first assume $b^{y_{1}} \geq 86$. From (3.4) and (3.5), this enables us to suppose that

$$
x_{2} \geq 43 x_{1} .
$$

Applying Lemma 5.1 and the trivial inequality $G(n) \geq 1$, we have

$$
|P|<a^{x_{1}(m-\delta)} \frac{4 \sqrt{2}}{3 \pi} \cdot 4^{m}
$$

and hence

Since

$$
\frac{b^{3 y_{1} m}}{\left|c_{2}\right||P|}>\frac{3 \pi}{\sqrt{2}}\left(\frac{b^{3 y_{1}}}{4 a^{x_{1}}}\right)^{m} \max \left\{a^{x_{2}}, b^{y_{2}}\right\}^{-1 / 2} .
$$

it follows that

$$
m=\frac{x_{2}-\alpha}{3 x_{1}},
$$

$$
m>\frac{x_{2}}{3 x_{1}}-1
$$

and so, together with $b^{y_{1}}>\frac{15}{16} a^{x_{1}}$, we have

$$
\frac{b^{3 y_{1} m}}{\left|c_{2}\right||P|}>\frac{3 \pi}{\sqrt{2}}\left(15^{3} 2^{-14} a^{2 x_{1}}\right)^{\frac{x_{2}}{3 x_{1}}-1} \max \left\{a^{x_{2}}, b^{y_{2}}\right\}^{-1 / 2},
$$


whence

$$
\frac{b^{3 y_{1} m}}{\left|c_{2}\right||P|}>\frac{8192}{1125} \pi \sqrt{2}\left(15^{3} 2^{-14}\right)^{\frac{x_{2}}{3 x_{1}}} a^{2 x_{2} / 3-2 x_{1}} \max \left\{a^{x_{2}}, b^{y_{2}}\right\}^{-1 / 2} .
$$

From (2.2) and the fact that $15^{3} 2^{-14}>\frac{1}{5}$, we thus have

$$
\frac{b^{3 y_{1} m}}{\left|c_{2}\right||P|}>\frac{2048}{1125} \pi \sqrt{30} \cdot\left(\frac{a^{\frac{1}{2}-\frac{6 x_{1}}{x_{2}}}}{5^{1 / x_{1}}}\right)^{x_{2} / 3} .
$$

Inequality (6.5) and the fact that $b^{y_{1}} \geq 86$ (whereby $a^{x_{1}} \geq 87$ ) thus imply

$$
a^{\frac{1}{2}-\frac{6 x_{1}}{x_{2}}}<5^{1 / x_{1}}
$$

and so

$$
\frac{b^{3 y_{1} m}}{\left|c_{2}\right||P|}>\frac{2048}{1125} \pi \sqrt{30}
$$

which yields (6.4).

To treat the cases where $b^{y_{1}} \leq 85$, we note that inequality (6.7) (and hence (6.4)) follows as before, from (3.4), unless we have either $16 \leq b^{y_{1}} \leq 36$ and $a^{x_{1}}=b^{y_{1}}+1$, or

$$
\left(a, x_{1}, b, y_{1}\right)=(2,6,63,1),(65,1,2,6),(66,1,2,6),(83,1,3,4) .
$$

If $b^{y_{1}} \geq 25$, then we have, in each case, (6.7) and hence (6.4), unless $x_{2} \leq 996$. For each $(a, b)$ under consideration, we compute the initial terms in the simple continued fraction expansion to $\frac{\log a}{\log b}$ and check that, in each case, convergents $p_{s} / q_{s}$ with $x_{1}<p_{s} \leq 996$ have corresponding partial quotients $a_{s+1}$ violating (3.6).

To treat the cases $16 \leq b^{y_{1}} \leq 24$, we argue as previously only with the trivial lower bound upon $G(n)$ replaced by that of Proposition 5.2. After a little work, we deduce the inequality

$$
\frac{b^{3 y_{1} m}}{\left|c_{2}\right||P|}>0.087(0.319)^{\frac{x_{2}}{3 x_{1}}} a^{x_{2} / 6-2 x_{1}} .
$$

In every case, this implies (6.4), unless $x_{2} \leq 158$. Again, examining the simple continued fraction expansions to $\frac{\log a}{\log b}$ for $a=b+1$ and $17 \leq b \leq 23$, and $(a, b)=(17,2),(5,24)$, we find that all convergents $p_{s} / q_{s}$ with $x_{1}<p_{s} \leq 158$ have corresponding partial quotients $a_{s+1}$ which contradict (3.6).

From inequalities (6.3) and (6.4), we thus have

$$
\frac{30 b^{3 y_{1} m}}{31|E|}<M_{1}=a^{\alpha} \leq a^{3 x_{1}-1} .
$$

Since

$$
|E|<\frac{4}{3 \sqrt{2} \pi} G(n)^{-1} c_{1}^{1-2 \delta} a^{x_{1}(\delta-1)}\left(16 c_{1}^{2} a^{2 x_{1}}\right)^{m},
$$

it follows from Proposition 5.2 that

$$
\left(\frac{1.5498 b^{3 y_{1}}}{16 c_{1}^{2} a^{2 x_{1}}}\right)^{m}<112 a^{(2+\delta) x_{1}-1} c_{1}^{1-2 \delta} .
$$

Now

$$
c_{1}=\frac{1}{4} a^{\theta x_{1}} \quad \text { where } \quad 0<\theta<1 / 2
$$


and hence we have

$$
\left(\frac{1.5498 b^{3 y_{1}}}{a^{(2+2 \theta) x_{1}}}\right)^{m}<112 \cdot 2^{4 \delta-2} a^{(2+\delta+\theta-2 \theta \delta) x_{1}-1}<448 a^{(3-\theta) x_{1}-1} .
$$

Again the fact that $b^{y_{1}}>\frac{15}{16} a^{x_{1}}$ yields

$$
\left(1.2769 a^{(1-2 \theta) x_{1}}\right)^{m}<448 a^{(3-\theta) x_{1}-1}
$$

and so, since $0<\theta<1 / 2$ and $a^{x_{1}}<\frac{16}{15} b^{y_{1}}$,

$$
m<4.1 \cdot \log \left(448 a^{3 x_{1}-1}\right)<25.9+12.3 \log \left(b^{y_{1}}\right)-4.1 \log a .
$$

On the other hand, from (3.5) and (6.6),

$$
m>\frac{\sqrt{15} b^{y_{1} / 2} \log a}{3 y_{1}}-\frac{5}{3},
$$

whence, with (6.8),

$$
\frac{b^{y_{1} / 2} \log a}{y_{1}}<21.4+9.6 \log \left(b^{y_{1}}\right)-3.1 \log a .
$$

This inequality provides an immediate contradiction for suitably large $b^{y_{1}}$ (and hence for all but finitely many quadruples $\left.\left(a, x_{1}, b, y_{1}\right)\right)$. We will treat these exceptions in the next section, completing the proof of Theorem 1.1.

\section{Computations}

Let us first dispense with the possibility that $\min \left\{x_{1}, y_{1}\right\}>1$. A short computation reveals that there are exactly 122 quadruples $\left(a, x_{1}, b, y_{1}\right)$ with $\min \left\{x_{1}, y_{1}\right\} \geq 2$ and

$$
b^{y_{1}}<a^{x_{1}} \leq 10^{8},
$$

satisfying (1.2).

From inequality (6.9), since $a \geq 2$, we may check that, if $y_{1}=2$, then necessarily $b \leq 385$, and, more generally

\begin{tabular}{|cc|cc|}
\hline$y_{1}=2$ & $b \leq 385$ & $y_{1}=7$ & $b \leq 7$ \\
$y_{1}=3$ & $b \leq 72$ & $y_{1}=8$ & $b \leq 6$ \\
$y_{1}=4$ & $b \leq 29$ & $y_{1}=9$ & $b \leq 5$ \\
$y_{1}=5$ & $b \leq 16$ & $10 \leq y_{1} \leq 15$ & $b \leq 3$ \\
$y_{1}=6$ & $b \leq 11$ & $16 \leq y_{1} \leq 25$ & $b=2$ \\
\hline
\end{tabular}

while, if $y_{1} \geq 26$, we have $b<2$, a contradiction. From (1.2), the inequalities in (7.1) thus obtain and it is therefore easy to check that the only quadruples satisfying the above bounds upon $y_{1}$ and $b$, together with (1.2), are

$$
\left(a, x_{1}, b, y_{1}\right)=(13,3,3,7),(56,2,5,5),(15,3,58,2),(2,15,181,2),(2,17,362,2) .
$$

To treat these remaining quadruples, in each case, we begin by noting that, from (6.8), $m \leq 167$. Inequality (6.6) and the fact that $x_{1} \leq 17$ thus imply that

$$
x_{2} \leq 8567 \text {. }
$$

For each of our five cases, as in the preceding section, we compute some initial terms in the infinite simple continued fraction expansion to $\frac{\log b}{\log a}$ via Maple 9.5. Since $x_{2}$ and $y_{2}$ are coprime, $x_{2}$ is the numerator of a convergent in such an expansion, say 
$x_{2}=p_{s}$. In each case, there are fewer than 5 convergents for which $x_{1}<p_{s} \leq 8567$; in no case does $a_{s+1}$ satisfy (3.6).

We may thus suppose

$$
\min \left\{x_{1}, y_{1}\right\}=1 \text {. }
$$

Let us begin by assuming that $x_{1}=1$. It follows that $a>b^{y_{1}}$ and hence we may replace (6.9) with the simpler

$$
b^{y_{1} / 2} \log b<21.4+96.5 \log \left(b^{y_{1}}\right),
$$

which implies the inequalities

\begin{tabular}{|cc|cc|}
\hline$y_{1}=1$ & $b \leq 120$ & $y_{1}=4$ & $b \leq 6$ \\
$y_{1}=2$ & $b \leq 20$ & $5 \leq y_{1} \leq 7$ & $b \leq 3$ \\
$y_{1}=3$ & $b \leq 7$ & $8 \leq y_{1} \leq 13$ & $b=2$ \\
\hline
\end{tabular}

We consider $a=b^{y_{1}}+t$ where, from (1.2),

$$
1 \leq t<\frac{\sqrt{1+64 b^{y_{1}}}+1}{32} .
$$

Since we omit perfect powers for $a$ and $b$, this leaves us with precisely 306 triples $\left(a, b, y_{1}\right)$. Combining (6.8) and (6.6), we thus have that $x_{2}=p_{s}, y_{2}=q_{s}$ for a convergent $p_{s} / q_{s}$ in the simple continued fraction expansion to $\frac{\log b}{\log a}$, satisfying

$$
1<p_{s}<77.1+24.6 \log a .
$$

A simple calculation reveals that none of these convergents have corresponding $a_{s+1}$ satisfying (3.6).

Finally, let us suppose that $y_{1}=1$ (and, from the preceding work, that $x_{1} \geq 2$ ). If $a=2$ then from (6.9), we have $b \leq 28913$ and so, via (1.2), $x_{1} \leq 14$. Similarly, for larger values of $a$, we may conclude as follows :

\begin{tabular}{|cc|cc|}
\hline$a=2$ & $x_{1} \leq 14$ & $a=6$ & $x_{1} \leq 4$ \\
$a=3$ & $x_{1} \leq 8$ & $a=7,10$ & $x_{1} \leq 3$ \\
$a=5$ & $x_{1} \leq 5$ & $11 \leq a \leq 22$ & $x_{1}=2$ \\
\hline
\end{tabular}

If $a \geq 23$, we contradict $x_{1} \geq 2$. For each pair $\left(a, x_{1}\right)$, we consider $b=a^{x_{1}}-t$, where

$$
1 \leq t<\frac{1}{4} a^{x_{1} / 2} .
$$

Once again, (6.8) and (6.6) imply the existence of a convergent $p_{s} / q_{s}$ in the simple continued fraction expansion to $\frac{\log b}{\log a}$ with

$$
x_{1}<p_{s}<12.3 \cdot \log \left(a^{3 x_{1}-1}\right)+3 x_{1}-1
$$

and, via (3.6), corresponding partial quotient $a_{s+1}$ satisfying

$$
a_{s+1}>\frac{\sqrt{15} b^{q_{s} / 2} \log a}{2 q_{s}}-2 .
$$

A short calculation with Maple 9.5 verifies that this does not occur, completing the proof of Theorem 1.1.

\section{Acknowledgments}

The author would like to thank Michael Filaseta for numerous helpful discussions. 


\section{REFERENCES}

[1] M. Bennett, Fractional parts of powers of rational numbers, Math. Proc. Cambridge Philos. Soc. 114 (1993), no. 2, 191-201.

[2] M. Bennett, On some exponential equations of S. S. Pillai, Canad. J. Math. 53 (2001), 897-922.

[3] F. Beukers, Fractional parts of powers of rationals, Math. Proc. Cambridge Philos. Soc. 90 (1981), no. 1, 13-20.

[4] J.W.S. Cassels, On the equation $a^{x}-b^{y}=1$, Amer. J. Math. 75 (1953), 159-162.

[5] J.W.S. Cassels, On the equation $a^{x}-b^{y}=1$. II, Proc. Cambridge Philos. Soc. 56 (1960), 97-103.

[6] W.J. Ellison, On a theorem of S. Sivasankaranarayana Pillai, Séminaire de Théorie des Nombres, 1970-1971 (Univ. Bordeaux I, Talence), Exp. No. 12, 10 pp. Lab. Théorie des Nombres, Centre Nat. Recherche Sci., Talence, 1971.

[7] A. Herschfeld, The equation $2^{x}-3^{y}=d$, Bull. Amer. Math. Soc. 42 [1936], 231-234.

[8] A.Y. Khinchin, Continued Fractions, P. Noordhoff Ltd., Groningen, 1963, 3rd edition.

[9] M. Laurent, M. Mignotte and Y. Nesterenko, Formes linéaires en deux logarithmes et déterminants d'interpolation, J. Number Theory 55 (1995), 285-321.

[10] W. J. LeVeque, On the equation $a^{x}-b^{y}=1$, Amer. J. Math. 74 (1952), 325-331.

[11] P. Mihăilescu, Primary cyclotomic units and a proof of Catalan's conjecture, J. Reine Angew. Math. 572 (2004), 167-195.

[12] S.S. Pillai, On the inequality $0<a^{x}-b^{y} \leq n$, J. Indian Math. Soc. 19 (1931), 1-11.

[13] S.S. Pillai, On $a^{x}-b^{y}=c$, J. Indian Math. Soc. (N.S.) 2 (1936), 119-122, and 215.

[14] S.S. Pillai, On $a^{X}-b^{Y}=b^{y} \pm a^{x}$, J. Indian Math. Soc. (N.S.) 8 (1944), 10-13.

[15] S.S. Pillai, On the equation $2^{x}-3^{y}=2^{X}+3^{Y}$, Bull. Calcutta Math. Soc. 37 (1945), 18-20.

[16] P. Ribenboim, Catalan's Conjecture, Academic Press, London, 1994.

[17] R. Scott and R. Styer, On the generalized Pillai equation $\pm a^{x} \pm b^{y}=c$, J. Number Theory, to appear.

Department of Mathematics, University of British Columbia, Vancouver, B.C. V6T $1 \mathrm{Z2}$

E-mail address: bennett@math.ubc.ca 